\title{
Crianças com Dificuldades Escolares Atendidas em Programa de Suporte Psicopedagógico na Escola: Avaliação do Autoconceito ${ }^{1}$
}

\author{
Cynthia Barroso Okano ${ }^{23}$ \\ Sonia Regina Loureiro \\ Maria Beatriz Martins Linhares \\ Edna Maria Marturano \\ Universidade de São Paulo, Ribeirão Preto
}

\begin{abstract}
Resumo
Objetivou-se avaliar o autoconceito de 40 crianças de ambos os sexos, na faixa etária de 7 a 10 anos, alunos de $1^{\mathrm{a}}$ e $2^{\mathrm{a}}$ série de uma escola da rede pública do município de Uberaba-MG, com nível intelectual pelo menos médio inferior, divididas em dois grupos: o G1 reuniu 20 crianças com dificuldades de aprendizagem escolar que freqüentam, além do ensino regular, um programa complementar denominado Ensino Alternativo e o G2, por sua vez, foi composto por 20 crianças sem dificuldades escolares freqüentando o ensino regular com bom rendimento. Os instrumentos utilizados foram: Matrizes Progressivas Coloridas - Raven Infantil - Escala Especial e Escala Infantil Piers-Harris de Autoconceito. Observou-se que as crianças do G1 apresentaram tanto o escore de autoconceito global quanto os escores de status intelectual e popularidade significativamente menor do que as crianças do G2.

Palauras-chave: Autoconceito; desempenho escolar; dificuldade de aprendizagem/criança.
\end{abstract}

Children with Learning Difficulties Attending a Psychopedagogic School Program: Evaluation of Self-Concept

\begin{abstract}
The objective of the present study was to assess the self-concept of 40 children of both sexes aged 7 to 10 years, enrolled in $1^{\text {st }}$ and $2^{\text {nd }}$ grade in a public school in the municipality of Uberaba, MG, of at least medium low intellectual level, divided into two groups: G1, consisting of 20 children with learning difficulties who were enrolled in a complementary program denoted Alternative Teaching in addition to the regular school program, and G2, consisting of 20 children with no learning difficulties, enrolled in a regular school program with good performance. The instruments used were: Progressive Color Matrices - Childhood Raven Special Scale and the Piers-Harris Children's Self-Concept Scale-Revised Manual. G1 children were found to have significantly lower overall self-concept and intellectual status scores, as well as significantly lower popularity than G2 children.

Keywords: Self-concept; school performance; learning difficulties/children.
\end{abstract}

A identificação das dificuldades de aprendizagem apresentadas por escolares vem crescendo, embora há muitos anos se reconheça à relevância de tais problemas. Neste contexto, novas concepções sobre o processo de ensino e aprendizagem vêm reforçando a importância da influência das variáveis internas como as escolhas, crenças, expectativas e emoções, tanto daqueles que ensinam como daqueles que aprendem (Boruchovitch, 1994). De acordo com os dados da UNESCO a retenção escolar no Brasil está entre as maiores da América Latina (Estado de São Paulo, 1997), sendo o percentual de repetência na primeira série do $1^{\circ}$ grau de aproximadamente $54 \%$, o que contribui para o atraso escolar e como conseqüência, para a evasão escolar.

Os efeitos adversos do fracasso escolar, quando a criança não desenvolve sua capacidade produtiva, têm apontado para a existência de relação entre dificuldades de

\footnotetext{
${ }^{1}$ Apoio - Projeto Integrado CNPq - Projeto Temático FAPESP.

${ }^{2}$ Este estudo foi baseado na dissertação de mestrado da primeira autora, realizada sob a supervisão da segunda autora, apresentada ao Programa de Pós-graduação em Psicologia.

${ }^{3}$ Endereço para correspondência: Av. Santos Dumont, 1520, 38050 400, Uberaba, MG. Fone/ Fax: (34) 33147777.E-mail:c.okano@terra.com.br
}

aprendizagem e baixa auto-estima, aceitação e popularidade perante os colegas (Linhares, Parreira, Marturano \& Sant'Anna, 1993).

Estudos empíricos e de revisão desde os anos 1970 (AbuHilal, 2000; Ashman \& Van Kraayenoord, 1998; Bloom, 1976; Chapman, 1988; Chapman \& Boersma, 1991; Covington \& Omelich, 1979; Hamachek, 1995; Harter \& Pike, 1984; Purkey, 1970) têm assinalado de forma sistemática correlações positivas entre autoconceito e o desempenho acadêmico. De forma geral, pode se dizer que os referidos autores partilham da afirmativa que conhecimentos e sentimentos positivos em relação a si próprio repercutem no bom funcionamento individual, na motivação e na forma como os indivíduos respondem às demandas da aprendizagem.

Os anos iniciais de escolarização parecem ser cruciais, no que diz respeito ao aprender a ler. Chapman e Tunner (1997) ao examinarem a interação entre autoconceito e o início da aquisição da leitura, verificam que as experiências de aquisições positivas de leitura, no início da escolarização, mostraram-se associadas ao desenvolvimento de autoconceito mais positivo nos primeiros dois anos e meio de escolarização 
formal. Observaram também neste período os efeitos negativos sobre o autoconceito das crianças que experimentaram dificuldades iniciais na aprendizagem da leitura.

Além de as crianças se auto-avaliarem, a escola também as avalia, tendendo a enfatizar as comparações sociais com base no rendimento escolar. Neste sentido, Renick e Harter (1989) relataram que o processo de comparação social é de grande importância na formação da autopercepção dos estudantes com dificuldades de aprendizagem no que se refere à competência acadêmica.

A dificuldade escolar pode gerar um "círculo vicioso do fracasso", ou seja, quanto mais a criança se sente inferiorizada, mais ela estará suscetível ao insucesso, e menos poderá obter aprovação a partir de seu desempenho (Linhares \& cols., 1993).

O manejo das dificuldades de aprendizagem no ambiente escolar não se constitui em tarefa fácil, e muitas vezes, a alternativa dada envolve a colocação das crianças em programas especiais de ensino como o proposto para as salas de reforço ou de recuperação paralela, destinadas a alunos com dificuldades não superadas no cotidiano escolar. Os programas de reforço, em nosso meio, a princípio se apresentam como uma proposta que visa contribuir para o bom desenvolvimento escolar, contudo carecem de estudos sistemáticos que demonstrem a sua eficácia no que diz respeito aos aspectos psicológicos de crianças com dificuldade de aprendizagem.

Diversos estudos têm relatado que as crianças com dificuldades de aprendizagem têm autopercepção mais negativa sobre o seu próprio comportamento quando comparadas a crianças que têm rendimento satisfatório e quando comparadas àquelas que têm baixo rendimento, mas não são identificadas como tendo dificuldade de aprendizagem (Beltempo \& Achile, 1990; Clever, Bear \& Juvonen, 1992; Leondari, 1993; Jackson \& Bracken, 1998).

Lidar com o insucesso escolar, com o baixo rendimento, e com as múltiplas implicações para a auto-avaliação da criança, para a família, professores e comunidade constituise em tarefa complexa e desafiadora para a qual não se tem ainda uma resposta acabada e pronta, o que aponta para a necessidade de buscar alternativas que possam minimizar tal situação.

Neste contexto, foram implantados na rede pública municipal de Uberaba-MG, programas de atendimento destinados a crianças com dificuldade de aprendizagem (Ensino Alternativo) e/ou com falta de suporte familiar no horário complementar à escola (Centro Integrado de Ensino Municipal).

O programa Ensino Alternativo (1996) foi implantado com o objetivo de ajudar as crianças que ingressam no ensino fundamental a superarem as dificuldades iniciais de forma a favorecer-lhes a aprendizagem e o desenvolvimento.

Tal programa consiste em uma dinâmica pedagógica que acontece na própria escola, em horário complementar, destinado aos alunos com dificuldades, selecionados para atendimento através de um sistemático diagnóstico psicopedagógico que os identifica e prioriza para preenchimento das vagas disponíveis. Todo esse processo é realizado pelas professoras treinadas e supervisionadas por pedagogas das unidades escolares e por psicólogas da rede pública.

O presente trabalho tem por objetivo avaliar o autoconceito de um grupo de crianças com dificuldades de aprendizagem escolar, que freqüentam o ensino regular associado a um programa de suporte psicopedagógico na escola (Ensino Alternativo), comparado a outro grupo formado por crianças sem dificuldades escolares que freqüentam apenas o ensino regular.

\section{Método}

\section{Caracterização do Contexto da Pesquisa}

A presente pesquisa foi desenvolvida na Escola Municipal "Professor Paulo Rodrigues" de Uberaba-MG.

Nesta escola são desenvolvidos dois programas de apoio aos escolares, a saber: o CIEM (Centro Integrado de Ensino Municipal) e o Ensino Alternativo.

A Escola Municipal "Professor Paulo Rodrigues" conta com duas classes de Ensino Alternativo, sendo uma pela manhã e outra à tarde. No ano de 1998, quando da coleta de dados do presente estudo, freqüentaram este programa 40 crianças, atendidas em pequenos grupos por dois períodos semanais complementares aos horários em que freqüentam o ensino regular.

A inclusão das crianças no Ensino Alternativo é feita mediante o encaminhamento da professora do ensino regular e a realização de uma prova pedagógica preparada pela equipe de Coordenação Central. A condição essencial para a inclusão da criança no Ensino Alternativo é a presença de dificuldade de aprendizagem. A constituição dos pequenos grupos de atendimento leva em conta o desempenho das crianças na prova pedagógica e o nível de dificuldade demonstrado.

\section{Participantes}

Participaram do presente estudo 40 crianças de ambos os sexos, na faixa etária de 7 a 10 anos, alunos de $1^{\mathrm{a}}$ e $2^{\mathrm{a}}$ série de uma escola municipal de Uberaba-MG.

As crianças foram distribuídas em dois grupos:

Grupo 1, composto por 20 crianças com dificuldades de aprendizagem escolar, avaliadas por prova pedagógica, que freqüentavam o ensino regular associado ao programa complementar denominado Ensino Alternativo; 
Grupo 2, composto por 20 crianças, sem dificuldades escolares, segundo a indicação das professoras, que freqüentavam o ensino regular.

Foram excluídos do estudo, participantes que apresentavam histórias ou deficiências físicas e déficits sensoriais visíveis.

Para a seleção dos participantes procedeu-se inicialmente a avaliação intelectual, através das Matrizes Progressivas Coloridas de Raven - MPC - Escala Especial (normas brasileiras de Angelini, Alves, Custódio \& Duarte, 1987), de 99 alunos da $1^{a}$ e $2^{a}$ série do ensino fundamental, sendo que 36 freqüentavam o ensino regular associado ao Ensino Alternativo e 63 freqüentavam apenas o ensino regular com e sem dificuldades escolares.

Com base na avaliação de nível intelectual das crianças que freqüentavam o Ensino Alternativo, foram selecionadas 31 crianças que apresentavam nível intelectual pelo menos médio inferior.

Considerando-se as características demográficas das 31 crianças que freqüentavam o Ensino Alternativo e que preencheram o critério quanto ao nível intelectual, foram então selecionadas as crianças sem dificuldades escolares, segundo a avaliação das professoras, que freqüentavam o ensino regular. Procedeu-se o balanceamento dos grupos quanto ao gênero (10 meninos e 10 meninas em cada grupo), idade (idade média: $\mathrm{G} 1=7$ anos e 3 meses e G2=7 anos e 9 meses) e escolaridade dos pais (média de 6 anos). Não foram observadas diferenças estatísticas significativas entre os grupos com relação a essas variáveis e ao nível intelectual avaliado pelo Raven. (Teste não-paramétrico de Kruskal Wallys, $p=0,05)$.

O projeto de pesquisa foi apreciado e aprovado pela diretoria da Escola Municipal "Professor Paulo Rodrigues". A participação das crianças no projeto foi voluntária, após solicitação aos pais do seu consentimento por escrito, mediante explicação relativa ao objetivo do trabalho, ao tipo de participação requerida, à ausência de prejuízos ou danos decorrentes da participação das crianças no projeto e o compromisso de encaminhamento a profissionais especializados caso fosse necessário. Foram ainda informados de que estávamos à disposição para os esclarecimentos que sentissem necessidade e que, a qualquer momento, poderiam desistir da sua participação, sem qualquer prejuízo para a criança.

\section{Instrumentos}

Matrizes Progressivas Coloridas - Raven Infantil- Escala Especial - avaliação do nível de inteligência das crianças normas brasileiras de Angelini e colaboradores (1987). Medida psicométrica de nível intelectual, incluída com o objetivo de controlar a interferência da variável déficit cognitivo, sendo excluídos os participantes com escores correspondentes à deficiência mental.

Escala Infantil Piers - Harris de Autoconceito "O que eu sinto sobre mim mesmo", avaliação do autoconceito das crianças. A escala original foi desenvolvida por Piers e Harris, (1984), traduzida e adaptada para o nosso meio por Jacob e Loureiro (1999). É composta por 80 afirmativas sobre como as crianças se sentem a respeito de si mesmas. A escala foi apresentada à criança solicitando-lhe que avaliasse se a sentença descrevia como ela se sentia em relação a si mesma na maioria das situações, devendo responder com "sim" ou "não", caso considerasse as sentenças verdadeiras ou falsas para si. A somatória das respostas com valor positivo correspondem ao escore total. O conjunto das afirmativas divide-se em agrupamentos relativos a seis categorias de autoconceito, a saber: comportamento, status intelectual e acadêmico, aparência física e atributos, ansiedade, popularidade, felicidade e satisfação.

\section{Procedimentos \\ Coleta de dados}

As crianças foram avaliadas pela primeira autora, em sessão individual, nas dependências da escola.

Inicialmente todas as crianças de $1^{\mathrm{a}}$ e $2^{\mathrm{a}}$ série que freqüentavam o programa de suporte psicopedagógico Ensino Alternativo foram avaliadas através do Raven Infantil-Escala Especial. Em seguida foram avaliadas as crianças que freqüentavam o ensino regular, indicadas pelas professoras como tendo bom desempenho escolar. As crianças responderam individualmente a prova conforme as recomendações da técnica, utilizando um tempo médio de aproximadamente 20 minutos. Com base nesta prova selecionaram-se as crianças para compor os dois grupos tendo como exigência percentil igual ou superior a 25.

Em uma segunda sessão individual, foi aplicada a Escala Infantil Piers - Harris de Autoconceito. Os itens da escala foram apresentados através de fita gravada na voz da aplicadora, evitando assim variações de leitura relativas às entonações e pausas. A criança podia ouvir o item tantas vezes quantas fossem necessárias, sempre através do gravador. A aplicadora, presente na situação, apenas assinalava a opção da criança na folha de respostas. Foram apresentados às crianças, 80 itens, com a instrução de responder sim, se a afirmativa fosse na maior parte das vezes verdadeira para ela, ou responder não, se a afirmativa fosse na maior parte das vezes falsa para ela. O tempo médio de aplicação foi de 20 minutos.

\section{Tratamento dos dados}

Os dados da avaliação do Raven foram analisados estatisticamente com a finalidade de verificar a homogeneidade dos grupos quanto ao nível intelectual. 
A codificação das subescalas da Escala Infantil PiersHarris de Autoconceito seguiu as recomendações do instrumento original (Piers \& Harris, 1984) e do estudo de tradução e adaptação realizado por Jacob e Loureiro (1999).

Em cada protocolo individual procedeu-se a quantificação relativa aos seguintes tópicos: Escore Total, somatória dos pontos em 80 itens, Escores relativos às escalas das seis classes específicas, a saber: comportamento, status intelectual e acadêmico, aparência física e atributos, ansiedade, popularidade, efelicidade e satisfação, além do Índice de Validade e Índice de Inconsistência.

Os dados codificados foram agrupados de acordo com o grupo de origem dos participantes, para que o tratamento estatístico pudesse ser realizado.

As respostas dos participantes dos dois grupos aos itens da Escala de Autoconceito foram analisadas através do Teste Qui-Quadrado visando comparar a proporção de participantes de cada grupo que responderam positivamente a cada item, e a identificação de diferenças estatisticamente significativas.

Em uma etapa posterior, as variáveis das técnicas foram relacionadas através do Coeficiente de Correlação nãoParamétrico de Spearman.
Nos testes estatísticos utilizados no presente estudo, considerou-se como critério para rejeição da hipótese de igualdade entre os grupos valores de $p=0,05$.

\section{Resultados}

Primeiramente, serão apresentados os dados relativos ao autoconceito quanto ao escore total. Em seguida, serão apresentados os dados relativos às seis categorias avaliadas, e os itens que diferenciaram os grupos 1 e 2 em nível de significância estatística. Finalmente, serão apresentadas as associações entre os resultados das avaliações realizadas através do Raven, e da Escala de Autoconceito, nos dois grupos de crianças respectivamente.

Os dados relativos à avaliação geral do autoconceito dos grupos 1 e 2, através da Escala Infantil Piers-Harris de Autoconceito, são apresentados na Tabela 1.

Observa-se na Tabela 1 que os participantes do grupo 2, crianças sem dificuldades escolares, obtiveram escores de autoconceito significativamente mais elevados quando comparados aos participantes do grupo 1, formado por crianças com dificuldades escolares que freqüentam o Ensino Alternativo. As primeiras portanto apresentam autoconceito global mais positivo do que as segundas.

Tabela 1

Autoconceito - Mediana e Comparação dos Grupos 1 e 2 relativas ao Escore Total e Índices Especificos

\begin{tabular}{lccc}
\hline Autoconceito & \multicolumn{2}{c}{ Mediana } & Comparação \\
\hline \multirow{2}{*}{ Escore total } & G1 & G2 & \\
Índice de inconsistência & 5,50 & 65,00 & \\
Índice de viés & 42,00 & 4,00 & G1 G2*** \\
& & & SD \\
\hline
\end{tabular}

Nota. Teste não-paramétrico de Kruskal-Wallys

$$
\begin{array}{r}
* * * p=0,001 \\
\text { SD - Sem diferença }
\end{array}
$$

\begin{tabular}{|c|c|c|c|}
\hline \multirow[t]{2}{*}{ Categorias } & \multicolumn{2}{|c|}{ Mediana } & \multirow[t]{2}{*}{ Comparação } \\
\hline & G1 & G2 & \\
\hline Comportamento & 11,50 & 14,00 & $\mathrm{G} 1<\mathrm{G} 2^{* *}$ \\
\hline Status intelectual & 12,00 & 14,00 & G1 < G2* \\
\hline Aparência & 10,00 & 10,50 & SD \\
\hline Ansiedade & 6,50 & 9,00 & G1 < G2* \\
\hline Popularidade & 6,50 & 8,50 & G1 < G2* \\
\hline Satisfação/felicidade & 8,00 & 9,00 & $\mathrm{SD}$ \\
\hline
\end{tabular}

Tabela 2

Nota. Teste não-paramétrico de Kruskal-Wallys

$$
\begin{aligned}
& * p=0,05 \\
& * * p=0,01 \\
& \text { SD }- \text { Sem diferença }
\end{aligned}
$$


Com relação aos índices de inconsistência e viés, não foram observadas diferenças com significância estatística entre os grupos estudados.

Os dados relativos aos escores das seis categorias de autoconceito apresentados pelos participantes dos grupos 1 e 2 são apresentados na Tabela 2.

Observa-se na Tabela 2 que em quatro das seis categorias avaliadas através da escala de autoconceito, os participantes do grupo 2, sem dificuldade escolar, apresentaram valores de mediana maiores do que os obtidos pelos participantes do grupo 1, com dificuldades escolares que freqüentam o Ensino Alternativo. As diferenças com significância estatística entre os grupos foram observadas nas categorias comportamento, status intelectual e acadêmico, ansiedade epopularidade. Esses dados mostram que as crianças sem dificuldades escolares (Grupo 2) apresentaram autoconceito mais positivo com relação ao comportamento, status intelectual e acadêmico e popularidade, apesar de se perceberem como mais ansiosas, comparativamente às crianças que apresentam dificuldades escolares (Grupo 1). Observou-se que os dois grupos de crianças não apresentaram diferenças estatisticamente significativas no que se refere às categorias aparência e felicidade.

Os itens que diferenciaram os grupos 1 e 2 com nível de significância estatística são apresentados na Tabela 3.

Observa-se na Tabela 3 que entre os 80 itens apresentados aos participantes, em 12 itens os resultados atingiram valores com significância estatística, revelando diferenças entre os dois grupos de crianças.

Em nove itens, uma menor porcentagem de participantes do grupo 1 (com dificuldades escolares) respondeu a estes de forma favorável a um autoconceito positivo, porém com valores inferiores aos observados nos participantes do grupo 2 (sem dificuldades escolares). Estes portanto apresentaram resultados sugestivos de um autoconceito mais positivo do que o do primeiro grupo. Especificamente em um item, cuja resposta de valoração positiva era o "Sim": "Eu sou bom em música", os participantes do grupo 1 apresentaram indicadores de um autoconceito mais positivo do que o grupo 2 .

Analisando-se as categorias de inclusão dos itens observase maior freqüência de itens relacionados ao comportamento $e$ status intelectual e acadêmico na diferenciação dos grupos. Apenas dois itens de outras categorias (ansiedade e popularidade) diferenciaram os grupos.

Quanto à categoria status intelectual e acadêmico, quatro dos 17 itens $(23,5 \%)$, incluídos nesta categoria diferenciaram os grupos. Nestes itens, o grupo 1, formado pelas crianças com dificuldades escolares, apresentou comparativamente aos participantes do grupo 2 , menor proporção de participantes que fizeram uma avaliação positiva de si no que diz respeito a sua capacidade, atenção e concentração, e conhecimentos gerais, assim como na presença de ansiedade quando chamado pela professora.

Tabela 3

Autoconceito - Itens ${ }^{1}$ que Diferenciaram os Grupos 1 e 2 com Significância Estatística

\begin{tabular}{|c|c|c|c|c|}
\hline Categoria & Item & G1\% & $\mathrm{G} 2 \%$ & Comparações \\
\hline Comportamento & 45. Eu não odeio escola & 70 & 100 & $\mathrm{G} 1<\mathrm{G} 2^{*}$ \\
\hline \multirow[t]{2}{*}{ (total de itens: 16) } & 56. Eu não entro em muitas brigas & 70 & 100 & $\mathrm{G} 1<\mathrm{G} 2 *$ \\
\hline & 62. Eu não sou atormentado em casa & 65 & 95 & $\mathrm{G} 1<\mathrm{G} 2 *$ \\
\hline $\begin{array}{l}\text { Status intelectual e } \\
\text { acadêmico }\end{array}$ & $\begin{array}{l}\text { 7. Eu não fico nervoso quando a professora me } \\
\text { chama }\end{array}$ & 65 & 100 & $\mathrm{G} 1<\mathrm{G} 2^{* *}$ \\
\hline \multirow[t]{3}{*}{ (total de itens:17) } & 53. Eu sei muitas coisas & 40 & 80 & $\mathrm{G} 1<\mathrm{G} 2 * *$ \\
\hline & $\begin{array}{l}\text { 26. Eu não sou lento em terminar meu trabalho } \\
\text { de escola }\end{array}$ & 35 & 70 & $\mathrm{G} 1<\mathrm{G} 2 *$ \\
\hline & 31. Na escola eu não fico no mundo da lua & 25 & 60 & $\mathrm{G} 1<\mathrm{G} 2^{* *}$ \\
\hline \multirow[t]{2}{*}{$\begin{array}{l}\text { Ansiedade } \\
\text { (total de itens:14) }\end{array}$} & $\begin{array}{l}\text { 7. Eu não fico nervoso quando a professora me } \\
\text { chama }\end{array}$ & 65 & 100 & $\mathrm{G} 1<\mathrm{G} 2 * *$ \\
\hline & 40. Eu não me sinto "por fora" das coisas & 20 & 75 & $\mathrm{G} 1<\mathrm{G} 2 * * *$ \\
\hline $\begin{array}{l}\text { Popularidade } \\
\text { (total de itens:12) }\end{array}$ & 40. Eu não me sinto "por fora" das coisas & 20 & 75 & $\mathrm{G} 1<\mathrm{G} 2 * * *$ \\
\hline Não categorizado & 64. Eu não sou desajeitado & 50 & 85 & $\mathrm{G} 1<\mathrm{G} 2 * *$ \\
\hline (total de itens:14) & 24. Eu sou bom em música & 75 & 40 & $\mathrm{G} 1>\mathrm{G} 2 * *$ \\
\hline
\end{tabular}

Total do Qui Quadrado

$* p \leq 0,05$

$* * p \leq 0,01$

$* * * p \leq 0,001$

${ }^{1}$ Considerou-se na apresentação dos itens a formulação dos mesmos acrescida da conotação valorativa positiva atribuída a eles conforme as recomendações da técnica. 
Dos 16 itens incluídos na categoria comportamento, 3 (18\%) diferenciaram os grupos 1 e 2 , indicando, uma autoavaliação das crianças do grupo 1 orientada para uma percepção de si negativa quanto ao seu comportamento na escola e em casa.

Já os itens incluídos na categoria ansiedade, também computados em status intelectual e acadêmico e popularidade, referem-se a autoavaliação de sentimentos relacionados a nervosismo e exclusão. Nenhum dos participantes do grupo 2 considerou-se nervoso quando chamado pela professora. Quanto ao item "Eu não me sinto por fora das coisas", também computado em popularidade, o grupo 2 , em uma maior porcentagem, não se considerou alheios aos acontecimentos, comparativamente ao Grupo 1.

Os itens "Eu não sou desajeitado" e "Eu sou bom em música" não se encontram categorizados em nenhuma das categorias, sendo computados apenas na contagem do escore total. Os participantes do grupo 2 perceberam-se como menos desajeitados, comparativamente aos participantes do grupo 1. Estes por sua vez se perceberam como tendo mais habilidade para a música comparativamente aos participantes do grupo 2.

Quanto às categorias aparência física e atributos (13 itens) e satisfação e felicidade (10 itens), não foi observada diferença significativa entre os grupos quanto a nenhum dos itens.

Os dados relativos à associação entre as variáveis dos grupos 1 e 2 são apresentados na Tabela 4.

A Tabela 4 mostra que, no grupo 1, constituído por crianças com dificuldades de aprendizagem escolar que freqüentam o Ensino Alternativo, observa-se que o nível cognitivo avaliado pelo Raven se correlacionou positivamente com o autoconceito relacionado a status intelectual.
Em relação ao autoconceito geral, notou-se correlações significativas do escore total com os escores parciais de autoconceito relativos a comportamento, status intelectual $e$ popularidade.

O autoconceito relacionado a status intelectuale acadêmico se correlacionou positivamente com o autoconceito relacionado à popularidade.

No grupo 2, crianças sem dificuldades escolares, observou-se que a avaliação de nível intelectual avaliado através do Raven, correlacionou-se positivamente com o autoconceito vinculado à popularidade.

O autoconceito geral correlacionou-se positivamente com as áreas autoconceito relacionado a comportamento, status intelectual, popularidade e ansiedade. Observou-se associações significativamente positivas entre autoconceito relacionado a comportamento com as áreas de status intelectual e ansiedade. Verificou-se correlações significativas positivas entre autoconceito relacionado a status intelectual e acadêmico com todas as demais áreas de autoconceito.

Observou-se associações positivas entre autoconceito relacionado à ansiedade e as demais áreas de autoconceito. $\mathrm{O}$ autoconceito relacionado à popularidade se correlacionou positivamente com o autoconceito geral e com as áreas de status intelectual e ansiedade.

\section{Discussão}

As crianças com dificuldades escolares do Grupo 1 se percebem com menor habilidade para aprender e com mais dificuldades comportamentais no sentido de ajustar-se às demandas do meio quando comparadas às crianças do Grupo

Tabela 4

\begin{tabular}{|c|c|c|c|c|c|c|c|}
\hline & & A & B & C & $\mathrm{D}$ & E & $\mathrm{F}$ \\
\hline \multirow[t]{2}{*}{ A } & G1 & 1,00 & 0,32 & 0,04 & $0,44^{*}$ & 0,08 & 0,09 \\
\hline & G2 & 1,00 & 0,21 & $-0,22$ & 0,05 & 0,26 & $0,44^{*}$ \\
\hline \multirow[t]{2}{*}{ B } & G1 & & 1,00 & $0,56^{*}$ & $0,77^{*}$ & 0,13 & $0,65 *$ \\
\hline & G2 & & 1,00 & $0,53^{*}$ & $0,86^{*}$ & $0,85 *$ & $0,88^{*}$ \\
\hline \multirow[t]{2}{*}{ C } & G1 & & & 1,00 & 0,19 & 0,24 & 0,30 \\
\hline & G2 & & & 1,00 & $0,60^{*}$ & $0,44^{*}$ & 0,26 \\
\hline \multirow[t]{2}{*}{$\mathrm{D}$} & G1 & & & & 1,00 & 0,00 & $0,45^{*}$ \\
\hline & G2 & & & & 1,00 & $0,59 *$ & $0,67^{*}$ \\
\hline \multirow[t]{2}{*}{$\mathrm{E}$} & G1 & & & & & 1,00 & 0,05 \\
\hline & G2 & & & & & 1,00 & $0,68^{*}$ \\
\hline \multirow[t]{2}{*}{$\mathrm{F}$} & G1 & & & & & & 1,00 \\
\hline & G2 & & & & & & 1,00 \\
\hline
\end{tabular}

Coeficiente de Correlação não paramétrica de Spearman

Legenda:

A- Raven

B- Autoconceito geral

C-Autoconceito/comportamento
D- Autoconceito/Status intelectual e acadêmico

E- Autoconceito/Ansiedade

F- Autoconceito/popularidade 
2. Estas percepções de autoconceito relacionadas a uma percepção negativa quanto às habilidades escolares apresentadas pelas crianças do Grupo 1 podem ser referidas ao que Renick e Harter (1989) destacam como efeito da comparação social. Os autores relatam em seus estudos que o processo de comparação social é de grande importância na formação da autopercepção dos estudantes com dificuldades escolares, no que se refere à competência acadêmica. Afirmam que estudantes com dificuldades escolares se percebem como menos competentes academicamente, quando se comparam com estudantes com rendimento satisfatório de suas salas de aula.

As percepções negativas, por parte do Grupo 1, no que se refere a comportamento e status intelectual podem estar relacionadas ao que Lindahl (1988) considera como o impacto continuado de situação de fracasso e desaprovações, o que possivelmente favorece a estas crianças sentirem-se inferiorizadas.

$\mathrm{O}$ autoconceito mais positivo do Grupo 2 nessas áreas também pode estar associado a uma maior autonomia e competência, variáveis estas consideradas por Deci, Hodges, Pierson e Tomassone (1992) como fatores motivacionais importantes para a aprendizagem.

Outro ponto a ser analisado diz respeito à maior popularidade das crianças do Grupo 2, possivelmente relacionado ao que Hamacheck (1995) destaca quanto aos estudantes com autoconceito mais positivo caracterizando-se como mais assertivos, sendo mais aceitos e respeitados nos grupos de pares.

Por outro lado, as crianças do Grupo 2 apresentaram mais indicadores de ansiedade comparativamente às crianças do Grupo 1, denotando possivelmente o esforço que empreendem para controlar-se e a preocupação em ser valorizado socialmente.

No que se refere às seis categorias de autoconceito avaliadas, não foram observadas diferenças estatisticamente significativas em relação à aparência e satisfação ou felicidade. Essas dimensões do autoconceito por sua vez não estão diretamente relacionadas com a habilidade acadêmica.

A percepção positiva das crianças do Grupo 1 quanto à habilidade para música pode ser considerada um recurso a ser aproveitado no sentido de preservar e favorecer o desenvolvimento destas crianças.

Em função do delineamento adotado selecionou-se para o estudo crianças com nível cognitivo pelo menos médio inferior. Nesse sentido foi possível assegurar que as crianças estudadas possuem recursos cognitivos para a aprendizagem podendo-se considerar as variações na avaliação do autoconceito associadas a variáveis afetivas, possivelmente relacionadas ao comportamento e à maturidade.

De modo geral, pode-se sugerir que as dificuldades de desempenho escolar das crianças do Grupo 1 estão associadas às vivências de fracasso relacionadas à diminuição do rendimento, o que pode estar reforçando sentimentos de inadequação e de baixa auto-estima, favorecendo outras situações conflituosas, dificultando a aquisição dos conteúdos formais e assim ampliando as dificuldades afetivas. Por outro lado, as crianças sem dificuldades escolares, com bom rendimento, segundo as professoras, mostram-se com autoconceito mais positivo e melhor aceitação pelos pares, mas denotam mais ansiedade sugerindo esforço para adaptar-se.

As crianças com dificuldades escolares que freqüentam o Ensino Alternativo concomitante e o ensino regular referiram autopercepções de dificuldades de comportamento e de se sentirem aceitas pelos outros, ou seja, colegas, professores e pela própria família. Pode-se supor que essas autopercepções estejam influenciando o que as crianças sentem com relação a si, afetando também o seu modo de viver e de agir. Perceberam-se como menos populares e capazes e como mais desajeitadas do que o seu grupo de pares, possivelmente em função das dificuldades escolares.

Estas autopercepções se contrapõe à apresentada pelas crianças sem dificuldades escolares. Possivelmente a boa capacidade de realização acadêmica dessas crianças está favorecendo-lhes essa auto-avaliação positiva, o que pode ter um efeito positivo para o seu desenvolvimento.

Observa-se assim a importância do desempenho acadêmico satisfatório para as crianças no que se refere ao autoconceito e também no que se refere à aceitação por parte dos colegas, professores, familiares e de si própria. A criança julga a si mesma de acordo com as observações que faz de si em comparação com os outros e das reações dos outros para com ela.

Possivelmente o autoconceito positivo por parte das crianças do Grupo 2 resulta também de outras experiências positivas com a vida familiar e escolar. Diversos trabalhos empíricos e de revisão (Abu-Hilal, 2000; Chapman \& Boersma, 1991; Hamachek, 1995) têm assinalado de forma sistemática, as relações entre autoconceito e o desempenho acadêmico, apontando que conhecimentos e sentimentos positivos em relação a si próprio repercutem no bom funcionamento individual, na motivação e na forma como os indivíduos respondem às demandas da aprendizagem, destacando-se a presença de correlação positiva entre autoconceito e desempenho nos diferentes estudos realizados com amostras diversas, selecionadas com base em variáveis distintas quanto à escolaridade, níveis sócio-econômicos, características étnicas e de habilidades dos participantes.

Vale destacar no presente estudo que em função do delineamento adotado, foi avaliada no Grupo 1, quase a totalidade das crianças que freqüentavam o Ensino Alternativo, não tendo sido controlada a variável tempo de permanência prévia no programa. Com relação ao Grupo 2 a avaliação de ausência de dificuldades escolares não foi sustentada por uma prova pedagógica específica, tendo por base o critério de indicação das professoras. Tal indicação pode ter sido influenciada não só pelo rendimento acadêmico dos alunos, mas também pelo comportamento dos mesmos em sala de aula. Contudo, os grupos foram considerados semelhantes quanto à idade e escolaridade dos pais, o que os torna comparáveis em aspectos relevantes. 
Quanto ao programa de suporte psicopedagógico na escola - Ensino Alternativo, conclui-se que as crianças com dificuldades escolares nele incluídas se percebem com baixa competência acadêmica, com dificuldades comportamentais e de aceitação pelos pares comparativamente às crianças sem dificuldades escolares. Tal dado chama atenção para a necessidade de valorização de outros princípios do Ensino Alternativo como o envolvimento da comunidade escolar e dos familiares dos alunos de modo a favorecer não só as habilidades acadêmicas, mas o desenvolvimento das habilidades sociais e de autoconceito, promovendo assim mecanismos de proteção ao desenvolvimento infantil.

\section{Referências}

Abu-Hilal, M. M. (2000). A strutural model for predicting mathematics achievement: It's relation with anxiety and self-concept in mathematics. Psychological Report, $86(3$ Pt 1), 835-847.

Angelini, A., Alves, I., Custódio, E. \& Duarte, W. (1987). Manual das matrižesprogressivas coloridas. São Paulo: Casa do Psicólogo.

Ashman, A. F. \& Van Kraayenoord, C. E. (1998). Educational characteristics of students with high or low self-concept. Psychology in the Schools, 35, 391-400.

Beltempo, J. \& Achile, P. A. (1990). The efect of special class placement on the selfconcept of children with learning disabilities. Child Study Journal, 20, 81-103.

Bloom, B. S. (1976). Human characteristics and school learning. New York, NY: McGraw Hill.

Boruchovitch, E. (1994). As variáveis psicológicas e o processo de aprendizagem: Uma contribuição para a psicologia escolar. Psicologia: Teoria e Pesquisa, 10, 129-139.

Briggs, D. C. (2000). A auto-estima do seu filho (W. Dutra, Trad). São Paulo: Martins Fontes. (Original publicado em 1986)

Chapman, J. W. (1988). Learning disabled children's self-concepts. Review of Educational Research, 58, 347-371.

Chapman, J. W. \& Boersma, F. J. (1991). Assessment of learning disabled students' academic self-concepts with the PASS: Findings from 15 years of research. Developmental Disabilities Bulletin, Special Issue, 19, 81-104.

Chapman, J. W. \& Tunner, W.E. (1997). A Longitudinal study of beginning reading achievement and reading self-concept. British Journal of Educational Psychology, 67, 279-291.
Clever, A., Bear, G. \& Juvonen, J. (1992). Discrepancies between competence and importance in self-perceptions of children in integrated classes. The Journal of Special Education, 26, 125-138.

Covington, M. V. \& Omelich, C. L. (1979). Are causal attributions causal? A path analysis of the cognitive model of achievement motivation. Journal of Personality and Social Psychology, 37, 1487-1504.

Deci, E. L., Hodges, R., Pierson, L. \& Tomassone, J. (1992). Autonomy and competence as motivational factors in students with learning disabilities and emotional handicaps. Journal of Learning Disabilities, 25, 457-471.

Ensino Alternativo (1996). Secretaria Municipal de Educação e Cultura de Uberaba. Uberaba, 01 de novembro.

Hamacheck, D. (1995). Self-Concept and school achievement: Interaction dynamics and a tool for assessing the self-concept component. Journal of Counseling and Development, 73, 419-425.

Harter, S. \& Pike, R. G. (1984). The pictorial scale of perceived competence and acceptance for young children. Child Development, 55, 1969-1983.

Jacob, A. V. (2001). O desempenho escolar e suas relações com autoconceito e auto-eficácia. Tese de Doutoramento, Curso de Pós-Graduação em Saúde Mental, Universidade de São Paulo. São Paulo, SP.

Jacob, A. V. \& Loureiro, S. R. (1999). O autoconceito e o desempenho escolar [Resumos]. Em Sociedade Brasileira de Psicologia (Org.), Anais, XXIX Reunião de Psicologia (p. 165). Campinas, SP: SBP.

Jackson, L. D. \& Bracken, B. A. (1998). Relationship between students' social status and global and domain - Specific Self - Concepts. Journal of School Pshichology, 36, 233-246.

Leondari, A. (1993). Comparability of self-concept among normal achievers, low achievers and children with learning difficulties. Educational Studies, 19, 357-371.

Lindahl, N. Z. (1988). Personalidade humana e cultura: Aplicações educacionais da teoria de Erik Erikson. Revista Brasileira de Estudos Pedagógicos, 69(163), 492-509.

Linhares, M. B. M., Parreira, V. L. C., Marturano, A. C. \& Sant' Anna, S. C. (1993). Caracterização dos motivos da procura de atendimento infantil em um serviço de psicopedagogia clínica. Medicina Ribeirão Preto, 26, 148-160.

Piers, E. V. \& Harris, D. B. (1984). Piers-Harris Children's Self-concept Scale-Revised Manual. Los Angeles: Counselor Psychological Tests, Western Psychological Services.

Purkey, W. W. (1970). Self concept and school achievement. Englewood Cliffs, NJ: Prentice Hall.

Renick, M. J. \& Harter, S. (1989). Impact of social comparisons on the developing selfperceptions of learning disabled students. Joumal of Educational Psychology, 81, 631-638.

Retenção está entre as maiores da A.L. (1997, 19 de outubro). Estado de São Paulo.

Recebido: 06/03/2003

$1^{a}$ Revisão: 06/06/2003

Última Revisão: 14/07/2003

Aceite Final: 21/07/2003

Sobre as autoras

Cyntia Barroso Okano é Mestre em Psicologia pela Faculdade de Filosofia, Ciências e Letras de Ribeirão Preto da Universidade de São Paulo. É Doutoranda em Saúde Mental pela Faculdade de Medicina de Ribeirão Preto da Universidade de São Paulo. É Especialista em Psicologia Clínica.

Sonia Regina Loureiro é Psicóloga, Professora Doutora da Faculdade de Medicina de Ribeirão Preto (USP) e docente nos Cursos de Pós-graduação em Ciências Médicas (Área: Saúde Mental) e Psicologia, na USP-RP. Coordena o Serviço de Psicoiagnóstico do Hospital de Clínicas de Ribeirão Preto, onde mantém atividades de formação de recursos humanos e de pesquisa com instrumentos e procedimentos de avaliação em diferentes contextos psicossociais.

Maria Beatriz Martins Linhares é Psicóloga, Especialista em Psicologia Clínica Infantil, Mestre em Educação Especial, Doutora em Psicologia Experimental. É Docente da Faculdade de Medicina da USPRP, orientadora no Curso de Pós-graduação em Saúde Mental (FMRP-USP) e Psicologia (FFCLRP-USP). É Coordenadora das seções de Psicologia do Desenvolvimento na área de Saúde e Psicologia Pediátrica do Serviço de Psicologia do Hospital das Clínicas da FMRP.

Edna Maria Marturano é Psicóloga, Professora Titular da FMRP-USP e docente nos Cursos de Pósgraduação em Ciências Médicas (Área: Saúde Mental) e Psicologia na USP-RP. Coordena o Ambulatório de Psicologia Infantil na Hospital de Clínicas de Rbeirão Preto, SP, onde mantém atividades de pesquisa, formação de recursos humanos e desenvolvimento de intervenções preventivas, focalizando crianças que vivem em situação de risco psicossocial e suas famílias. 